\title{
Age-related changes in elastographically determined strain of the facial fat compartments: a new frontier of research on face aging processes
}

\author{
Łukasz Paluch'1, Piotr Pietruski², Bartłomiej Kwiek ${ }^{3,4}$, Bartłomiej Noszczyk², Marcin Ambroziak²,3 \\ 'Department of Radiology, Gruca Orthopedic and Trauma Teaching Hospital, Medical Center of Postgraduate Education, Otwock, \\ Poland \\ 2Department of Plastic Surgery, Medical Center of Postgraduate Education, Orlowski Memorial Hospital, Warsaw, Poland \\ ${ }^{3}$ Ambroziak Clinic, Warsaw, Poland \\ ${ }^{4}$ Department of Dermatology and Immunodermatology, Warsaw Medical University, Warsaw, Poland
}

Adv Dermatol Allergol 2020; XXXVII (3): 353-359

DOI: https://doi.org/10.5114/ada.2018.79778

\begin{abstract}
Introduction: Our study goal was verification of shear-wave elastography (SWE) as an assessment tool enabling quantitative analysis of facial fat tissue elasticity, using the example of the deep medial cheek fat compartment (DMCFC), due to its major role in pseudoptosis etiology.

Aim: Furthermore, we determined the age-specific reference values for DMCFC elasticity and analyzed its correlation with body mass index (BMI) and DMCFC thickness.

Material and methods: The study included 89 female patients (age: 18-63 years, mean: $45.9 \pm 14.2$ years) with intact facial skin. Prior to the procedure, all participants were subjected to SWE of the DMCFC. Reference ranges for elastographic parameters were defined as \pm 2 standard deviations (SD), or estimated by means of ROC analysis. Results: The DMCFC elasticity correlated inversely with DMCFC thickness $(R=-0.292, p<0.001)$, age $(R=-0.838$, $p<0.001)$ and $\mathrm{BMI}$ of the study subjects $(R=-0.258, p=0.001)$. Age was found to be the only independent determinant of DMCFC elasticity on multiple linear regression analysis $(\beta=-0.837, p<0.001)$. The cut-off values for DMCFC elasticity estimated during ROC analysis provided excellent accuracy in distinguishing between women from various age categories, and to a large degree overlapped with the reference intervals defined as \pm 2 SD.

Conclusions: Shear-wave elastography enables quantitative evaluation of facial fat pad elasticity, creating a new frontier in research on age-related processes. The results indicate that elasticity of the DMCFC decreases significantly with age. Tissue elasticity might be an indirect indicator of metabolic and structural properties of facial adipose tissue and its extracellular matrix.
\end{abstract}

Key words: elastography, strain, deep medial fat pad, elasticity.

\section{Introduction}

In recent years, there has been a significant improvement in knowledge about facial fat compartments and their age-related changes. Subcutaneous fat was once believed to be a single uniform mass. However, recent studies indicate that this fat is divided into multiple compartments separated by a facial retaining system composed of fibrous fascial ligaments [1-4]. In general, fat pads can be categorized into superficial and deep compartments. In the midface region, the first group includes the nasolabial fat, the infraorbital fat, and the superficial medial cheek fat. The deep compartments, situated be- low the superficial musculoaponeurotic system (SMAS), include the sub-orbicularis oculi, buccal fat pad, deep lateral cheek pad, and deep medial cheek pad [1, 2, 5-8].

Findings from clinical observations and studies clearly indicate that various fat compartments undergo various age-related changes, with volume deflation being the main process. In addition, an inferior displacement along with increased distances between the fat pads is observed [1, 2, 7-9]. Volume loss does not have a global character, but is rather limited to the deep fat compartments supporting the overlying structures $[1,3$, $8,9]$. Some studies have even demonstrated that some

Address for correspondence: Marcin Ambroziak MD, Ambroziak Clinic, 13 Sikorskiego St, 02-758 Warsaw, Poland,

e-mail: marcin@klinikaambroziak.pl

Received: 15.10 .2018 , accepted: 24.10.2018. 
superficial facial fat seems to undergo hypertrophy in the elderly [10, 11]. The midfacial "pseudoptosis" theory suggests that selective atrophy of the deep medial cheek fat compartment (DMCFC) is the main factor contributing to a loss of anterior projection of nasolabial and nasojugal folds (sagging of the superficial compartments), inferior displacement of the lower lid (V-deformity) and unpleasant aesthetics of the palpebromalar groove, nasojugal fold, and tear trough [1, 7-12].

The increased knowledge of fat pads' anatomy and their age-related changes have altered the modern facial rejuvenation strategies, previously based on the lifting of the SMAS and skin along with fat compartments as one mass [1, 2, 8, 9, 13]. Nowadays, one can observe a trend of a shift towards specific compartment augmentation with a fat graft or filler as a primary procedure of choice for anterior midfacial projection restoration and improvement of pseudoptosis symptoms [2, 3, 7, 8, 12-14]. In this concept, DMCFC is considered to be the most important structure which should be addressed in the first place (Figure 1) [8, 13].

Regardless of multiple investigations on understanding facial physiologic age-related changes, there still is much to be done in terms of fat compartments. The formulated aging mechanism of those structures is mainly based on clinical observations and cadaveric dissections or imaging studies such as computed tomography (CT) or magnetic resonance imaging (MRI). Therefore, it should be considered a conjectural concept rather than a straightforward conclusion supported by quantitative

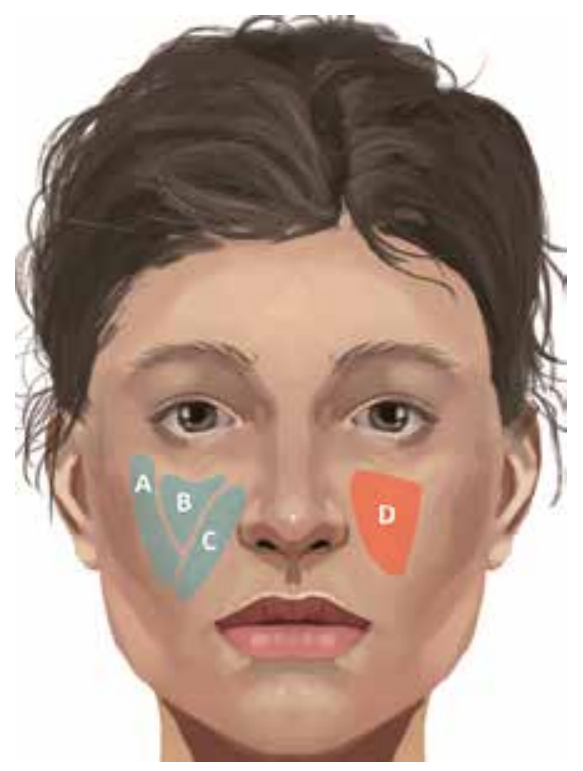

Figure 1. Superficial (blue color) and deep (red color) fat compartments crucial for midface region rejuvenation. A - Superficial lateral cheek fat compartment, B - superficial middle cheek fat compartment, $\mathrm{C}$ - superficial nasolabial fat compartment, D - deep medial cheek fat compartment data [8-10, 12]. Little is known about the internal structural characteristics of fat compartments and their differences among various facial regions and age groups. Such information could play a major role not only in better understanding of the aging process but also in facial rejuvenation results analysis and formulation of new patient-specific treatment algorithms. Some previous studies demonstrated that the age-related changes within soft tissues of the face can be documented by means of imaging studies such as MRI or CT $[15,16]$, but those methods are not widely available and generate substantial costs.

In our opinion, valuable structural characteristics of the facial fat compartments may be accurately determined with a less expensive and more accessible method, ultrasonographic shear wave elastography (SWE).

\section{Aim}

Our study aim was verification of the potential of this technology in the quantitative tissue elasticity assessment of facial fat, taking the example of the deep medial cheek fat pad, due to its major role in pseudoptosis etiology. Furthermore, we determined the age-specific reference values for DMCFC elasticity. Finally, the relationship of DMCFC elasticity with body mass index (BMI) and DMCFC thickness was analyzed.

\section{Material and methods}

The study, conducted at the University Department of Plastic Surgery and at a private plastic surgery and esthetic medicine clinic in Warsaw, included all consecutive female patients qualified for high-intensity focused ultrasound facial treatment. Only patients aged at least 18 years at the time of enrollment were qualified for the study. Women with visible scars or other skin lesions on the face, present or past history of connective tissue diseases, other autoimmune disorders, diseases of the skin and subcutaneous tissue and/or peripheral blood vessels, allergy or atopy, surgery or trauma involving the face, as well as active current and past smokers, were non-eligible for the study.

The study procedures were carried out in accordance with the Declaration of Helsinki. The protocol of the study was approved by the Institutional Bioethical Committee, and written informed consent was sought from all the subjects.

Prior to the laser treatment, all participants were subjected to ultrasonographic examination and elastography of the DMCFC. The examination was carried out with the patient in a supine position. Sonographic scans were obtained with a Toshiba iAplio 900 ultrasonograph with a 5-18 MHz transducer (Canon Medical Systems, Nasushiobara, Japan). During the examination, the face was covered with a hydrogel pad and a thick layer of gel. 


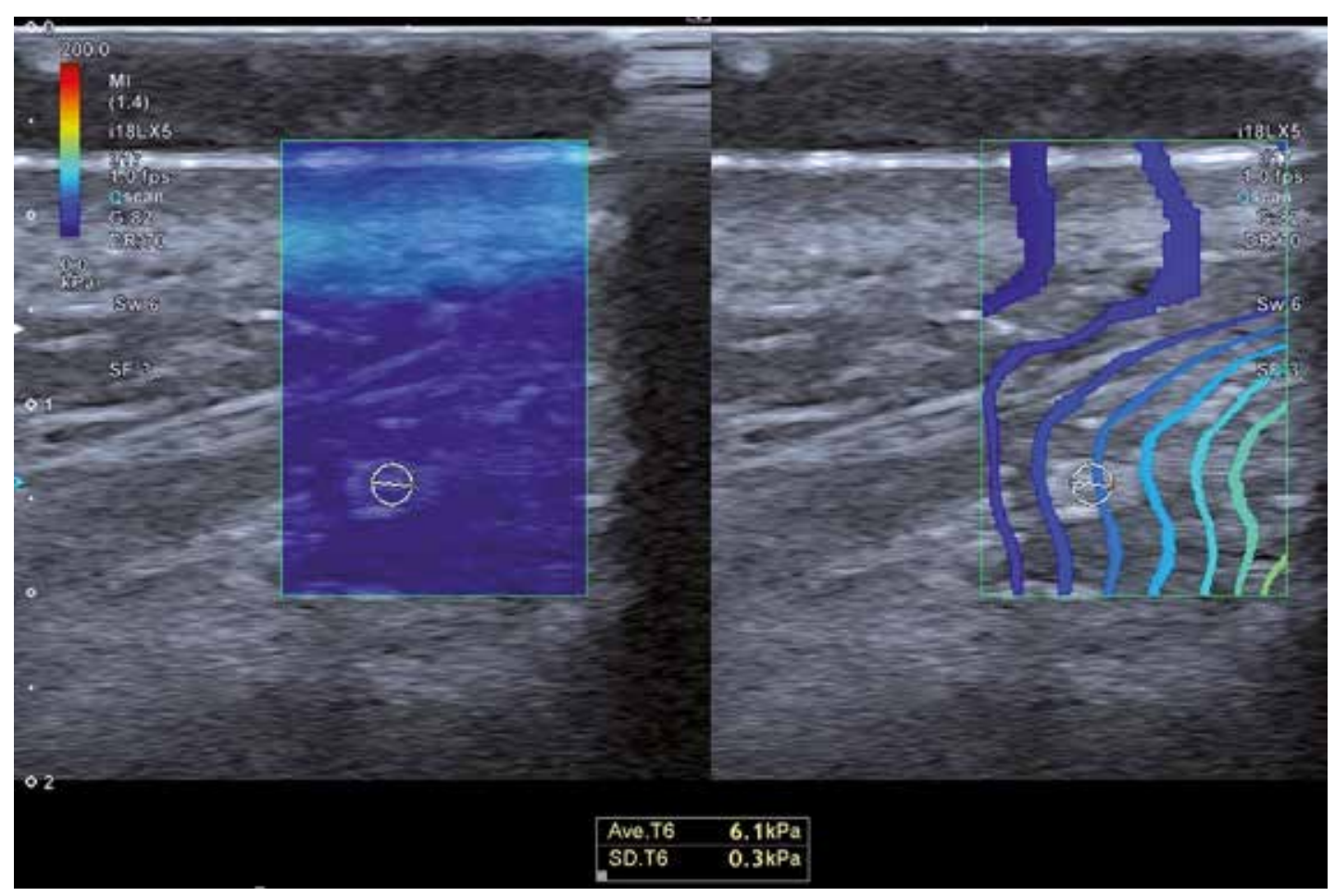

Figure 2. Representative elastographic image of DMCFC

The transducer was placed perpendicularly to the skin, and transverse scans were obtained. Upon visualization of the area of interest, the thickness of the DMCFC in millimeters was measured. Then, SWE was performed, after stabilizing the elastographic image. The region of interest (ROI) was placed in the center of the screen, to cover approximately $80 \%$ of the examined structure. Three measurements were taken for each ROI and the average result was recorded. The reference value for the elasticity modulus was set at $100 \mathrm{kPa}$. Representative elastographic images are presented in Figure 2.

\section{Statistical analysis}

Normal distribution of the study variables was verified with the Shapiro-Wilk test, and their statistical characteristics were presented as arithmetic means and their standard deviations (SDs), as well as medians and ranges. Power and direction of relationships between pairs of the variables were estimated on the basis of Spearman coefficients of rank correlation $(R)$. The effects of multiple predictors were analyzed using multiple regression analysis; the regression coefficient ( $\beta$ ) was calculated, along with its standard error (SE) and coefficient of determination for the regression model $\left(R^{2}\right)$. Intergroup comparisons were conducted with the Kruskal-Wallis test with Dunn post-hoc tests. Reference ranges for elastographic parameters were defined with two methods, as \pm 2 SD and on the basis of ROC analysis. During the latter, cut- off values of the elastographic parameters which optimally distinguished between various age categories were identified, along with their sensitivity, specificity, positive and negative predictive values (PPV and NPV, respectively) and the areas under ROC curves (AUC) with their 95\% confidence intervals $(95 \% \mathrm{Cls})$. All calculations were carried out with the Statistica 10 package (StatSoft, United States) with the threshold of statistical significance set at $p \leq 0.05$.

\section{Results}

\section{Characteristics of study participants}

The study included 89 women aged between 18 and 63 years (mean: $45.9 \pm 14.2$ years). The study group was divided into four age categories: women aged 39 years or younger ( $n=18,20 \%), 40$ - to 49 years old ( $n=28,31 \%$ ), 50 to 59 year old $(n=29,33 \%)$, and 60 years old or older $(n=14,16 \%)$. Descriptive statistics for the thickness and elasticity of the DMCFC, as well as other characteristics of the study participants, are presented in Table 1.

\section{Factors determining strain of the deep medial cheek fat compartment}

A strong significant inverse correlation was found between age of the study participants and DMCFC elasticity $(R=-0.838, p<0.001)$. Moreover, age showed a moderately strong correlation with DMCFC thickness 
Table 1. Basic characteristics of study participants and descriptive statistics for DMCFC thickness and strain

\begin{tabular}{lccccc}
\hline Parameter & Mean & SD & Median & Min. & Max. \\
\hline Age [years] & 45.9 & 14.2 & 49 & 18 & 67 \\
\hline BMI $\left[\mathrm{kg} / \mathrm{m}^{2}\right]$ & 24.76 & 3.22 & 24.68 & 18.59 & 33.98 \\
\hline $\begin{array}{l}\text { DMCFC } \\
\text { thickness }[\mathrm{mm}]\end{array}$ & 2.31 & 0.74 & 2.39 & 1.00 & 5.50 \\
\hline DMCFC strain $[\mathrm{kPa}]$ & 11.2 & 6.9 & 7.9 & 4.7 & 35.7 \\
\hline
\end{tabular}

Table 2. Results of multiple linear regression analysis, documenting the effects of age, BMI and DMCFC thickness on DMCFC elasticity

\begin{tabular}{lccc}
\hline Predictor & \multicolumn{3}{c}{ DMC $\left(R^{2}=0.754\right)$} \\
\cline { 2 - 4 } & $\beta$ & SE & $P$-value \\
\hline Age [years] & -0.837 & 0.040 & $<0.001$ \\
\hline BMI $\left[\mathrm{kg} / \mathrm{m}^{2}\right]$ & -0.050 & 0.039 & 0.197 \\
\hline DMCFC thickness $[\mathrm{mm}]$ & -0.058 & 0.039 & 0.144 \\
\hline
\end{tabular}

Table 3. Comparison of DMCFC elasticity (in $\mathrm{kPa}$ ) in participants from various age categories

\begin{tabular}{|c|c|c|c|c|c|c|c|c|c|c|}
\hline \multirow{2}{*}{$\begin{array}{l}\text { Age group } \\
\text { [years] }\end{array}$} & \multirow[t]{2}{*}{$N$} & \multirow{2}{*}{$\begin{array}{l}\text { Mean } \\
\text { strain }\end{array}$} & \multirow[t]{2}{*}{ SD } & \multirow[t]{2}{*}{ Median } & \multirow[t]{2}{*}{ Min. } & \multirow[t]{2}{*}{ Max. } & \multicolumn{4}{|c|}{$P$-values (post-hoc analysis) } \\
\hline & & & & & & & $<40$ years & $40-49$ years & $50-59$ years & $\geq 60$ years \\
\hline$<40$ & 18 & 22.4 & 5.8 & 23.2 & 13.0 & 35.7 & - & $<0.001$ & $<0.001$ & $<0.001$ \\
\hline $40-49$ & 28 & 11.6 & 3.4 & 11.0 & 6.0 & 18.3 & $<0.001$ & - & $<0.001$ & $<0.001$ \\
\hline $50-59$ & 29 & 6.4 & 0.9 & 6.3 & 4.7 & 8.7 & $<0.001$ & $<0.001$ & - & 0.679 \\
\hline$\geq 60$ & 14 & 5.8 & 0.6 & 5.0 & 5.0 & 7.0 & $<0.001$ & $<0.001$ & 0.679 & - \\
\hline
\end{tabular}

$<40$ years $v s .40-49$ years.

$(R=0.254, p=0.001)$. DMCFC elasticity correlated also inversely with $\mathrm{BMI}$ of the examined women $(R=-0.258$, $p=0.001)$. No statistically significant correlation was observed between BMI and DMCFC thickness $(R=0.103$, $p=0.171)$. Finally, a significant inverse correlation was documented between DMCFC thickness and elasticity $(R=-0.292, p<0.001)$. However, multiple linear regression analysis demonstrated that the age of the study participants was the only independent determinant of DMCFC elasticity among the three variables mentioned above (age, BMI, and DMCFC thickness) (Table 2).

During the next stage, we compared the elasticity of the DMCFC in women from various age categories. Participants from all age categories except from women aged 50-59 years and older than 60 years differed significantly in terms of DMCFC elasticity (Table 3 ).

\section{Reference values for the deep medial cheek fat compartment strain}

Considering the results presented above, we determined the reference values for DMCFC elasticity for three age categories: under 40 years, $41-49$ years, and above 50 years. The cut-off values for DMCFC elasticity estimated during ROC analysis provided excellent accuracy in distinguishing between women from various age categories (Figure 3). Furthermore, the reference values determined with this method to a large degree overlapped with the reference intervals defined as \pm 2 SD for a given age category (Table 4).

\section{Discussion}

Elastography, a sonographic technique to estimate tissue elasticity, was first implemented in clinical prac- tice in the 1990s [17]. Elastography measures the deformability of tissues caused by an external force, typically compression with an ultrasonographic transducer (strain elastography - SE), or the velocity of shear wave propagation within the tissue (shear wave elastography SWE) $[18,19]$. Both literature data and our experience indicate that SWE, measuring tissue elasticity in $\mathrm{kPa}$, is a more accurate and objective elastographic technique than SE $[19,20]$. Previous studies confirmed the applicability of elastography to many medical disciplines, primarily as a method to distinguish between normal and pathologically altered tissues [21]. However, a growing body of evidence suggests that elastography may also find application in esthetic medicine, as an instrument to examine the elasticity of the skin and adjacent structures [22-24].

The DMCFC lies between the periosteum of the maxilla and deep muscles, and therefore is under constant pressure, which may be one of the factors responsible for its relatively large atrophy with age. Due to its crucial role in age-related facial contour incongruity and importance in modern rejuvenation algorithms, we found it to be a perfect target for our preliminary study utilizing SWE technology.

Analysis of the results clearly showed that aging was associated with a statistically significant decrease in DMCFC elasticity; moreover, age turned out to be the only independent determinant of deep medial cheek fat compartment stiffness in the study group. To the best of our knowledge, there is no published research analyzing the elastographically determined elasticity of any facial fat compartment and its age-related changes; thus, we can only speculate about potential causes of this phenomenon. 

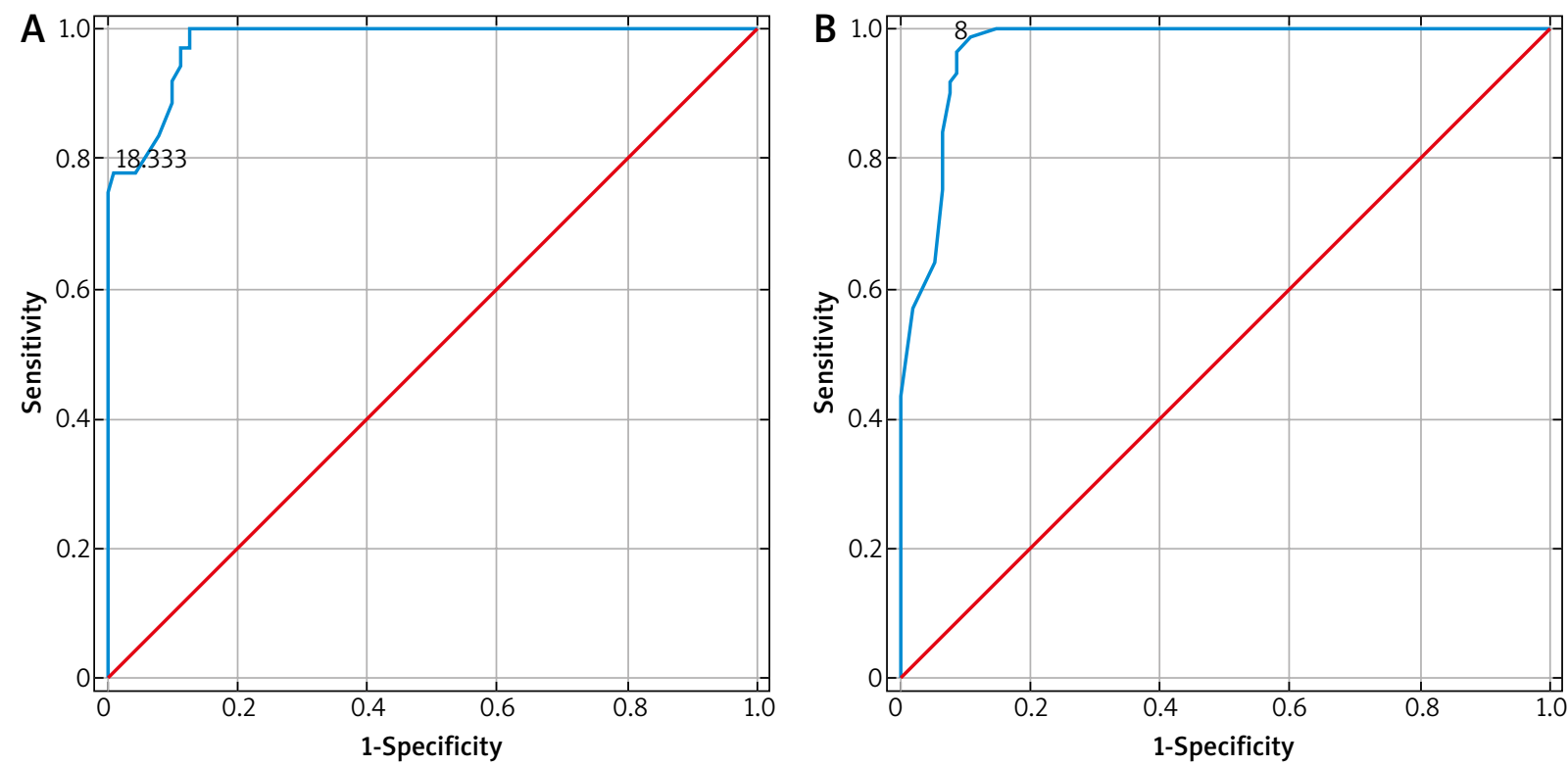

Figure 3. ROC curves illustrating the accuracy of age-specific cut-off values for DMCFC elasticity: (A) $>18.333 \mathrm{KPa}$ for women $<40$ years, (B) $<8 \mathrm{KPa}$ for women $\geq 50$ years

Table 4. Proposed reference ranges for DMCFC elasticity

\begin{tabular}{lccccccc}
\hline \multirow{2}{*}{$\begin{array}{l}\text { Age group } \\
\text { [years] }\end{array}$} & \multicolumn{2}{c}{ Reference value } & Sensitivity & Specificity & PPV & NPV & AUC (95\% Cl) \\
\cline { 2 - 5 } & \pm 2 SD $[\mathrm{kPa}]$ & ROC $[\mathrm{kPa}]$ & & & & & \\
\hline$<40$ & $11-34$ & $>18$ & 0.778 & 0.993 & 0.966 & 0.946 & $0.979(0.963-0.996)$ \\
\hline $40-49$ & $5-18$ & $8-19$ & - & - & - & - & - \\
\hline$\geq 50$ & $4-8$ & $<8$ & 0.965 & 0.913 & 0.912 & 0.966 & $0.970(0.947-0.993)$ \\
\hline
\end{tabular}

Probably, a key to the age-related decrease in DMCFC elasticity might be its microscopic structure and changes it undergoes with age. In line with the microscopic classification of subcutaneous fat, proposed by Ghassemi et al. [25], it represents a type 1 fat tissue with a loose linkage between the collagenous meshwork surrounding the adipocytes, the facial muscles, and the periosteum. The extracellular matrix of white adipose tissue, although sparse, plays an important active role in DMCFC morphology and physiologic processes [26]. Therefore, the primary cause of the hereby documented age-related decrease of the elasticity values of the fat pad might be degradation of the otherwise sparse collagen network in this tissue [27]. A similar age-progressive process, mediated by matrix metalloproteinases released from dermal keratinocytes, has already been documented in the case of type I collagen, the major structural protein of the skin [28]. Recent evidence suggests that aside from age, the activity of matrix metalloproteinases may also be stimulated by increased subcutaneous white adipose tissue $[29,30]$. However, unlike the amount of subcutaneous fat, the volume of the deep fat compartments does not seem to be BMI-dependent [31]; this was also confirmed in our study, which did not demonstrate a significant correlation between DMCFC thickness and BMI of the study participants. Nevertheless, we observed an inverse correlation between BMI and DMCFC elasticity, which suggests that an increase in body weight of the study participants might contribute to local or systemic changes in fat ultrastructure, like the mentioned activation of metalloproteinase activity and resultant degradation of the collagen network.

Taking into account the statistically significant correlations between participant age and DMCFC elasticity, we have defined its reference values for three age categories: < 40 years, 40 to 49 years, and 50 years and older. The appropriateness of such stratification was verified based on the intergroup comparisons of the elastographic parameters. The reference values were estimated with two methods, as \pm 2 SD and using ROC analysis. Importantly, both sets of reference values overlapped to a large degree, and the cut-off values of DMCFC elasticity estimated during ROC analysis provided excellent accuracy in distinguishing between women from various age categories. The hereby presented reference values may find application in esthetic medicine, e.g. to determine 
whether the DMCFC in a given patient is tight enough to be suitable for a specific rejuvenation procedure or to objectively evaluate the treatment results.

Overall, our study confirmed that SWE can be used to assess the elasticity of the facial fat compartment in a reliable, quantitative manner. This technology may be a valuable addition to the facial aging process research armamentarium. The elasticity of the fat pad might be a hallmark of metabolic and structural properties of adipose tissue and its extracellular matrix. Further studies, utilizing fat histological and volumetric analysis, are needed in order to confirm and fully understand this relation. Moreover, SWE might have clinical application in routine aesthetic medicine practice. Ability to evaluate fat compartments' structural characteristics enables objective analysis of facial rejuvenation results, eventually leading to improvements of treatment algorithms. Rapid assessment of patient fat compartments' strain value combined with the creation of a reference database of tissue elasticity of each significant fat compartment in a certain age group would allow treatment option personification, tailored to the specific need.

Our study was not free from potential limitations. The first of them was a relatively small patient sample size, especially in the context of age group analysis. Nevertheless, to the best of our knowledge, this was the only elastographic study of the facial fat pad that has been conducted thus far. Second, it cannot be excluded that the elastographic parameters determined in this study were influenced by some non-identified confounders. We tried to overcome this potential bias, enrolling solely patients who satisfied the most exhaustive list of inclusion and exclusion criteria. Finally, the hereby presented findings might be biased due to some drawbacks inherent to elastographic examination of a relatively thin layer of examined fat pad, located in close proximity to other tissues with higher values of Young's modulus, such as muscles and bones, or due to subjective selection of the ROI. We did our best to neutralize these potential limitations, using a high-frequency transducer and a hydrogel pad, reducing the diameter of the ROI and repeating each measurement in pursuit of better reproducibility. Finally, our work due to its preliminary character determined the elasticity reference values for only DMCFC. Future research will cover all significant superficial and deep facial fat compartments, providing an overall insight into tissue elasticity distribution among various facial regions, age groups and sexes.

\section{Conclusions}

Shear-wave elastography enables quantitative evaluation of facial fat pad elasticity, creating a new frontier of research into age-related processes. Our study results show that elastographically determined elasticity of the DMCFC decreases significantly with age. The elasticity of the fat pad might be an indirect indicator of metabolic and structural properties of facial adipose tissue and its extracellular matrix.

\section{Conflict of interest}

The authors declare no conflict of interest.

\section{References}

1. Rohrich RJ, Pessa JE, Ristow B. The youthful cheek and the deep medial fat compartment. Plast Reconstr Surg 2008; 121: 2107-12.

2. Rohrich RJ, Pessa JE. The fat compartments of the face: anatomy and clinical implications for cosmetic surgery. Plast Reconstr Surg 2007; 119: 2219-27.

3. Rohrich RJ, Pessa JE. The retaining system of the face: histologic evaluation of the septal boundaries of the subcutaneous fat compartments. Plast Reconstr Surg 2008; 121: 1804-9.

4. Schaverien MV, Pessa JE, Rohrich RJ. Vascularized membranes determine the anatomic boundaries of the subcutaneous fat compartments. Plast Reconstr Surg 2009; 123: 695-700.

5. Owsley JQ. Lifting the malar fat pad for correction of prominent nasolabial folds. Plast Reconstr Surg 1993; 91: 463-74.

6. Hamra ST. A study of the long-term effect of malar fat repositioning in face lift surgery: short-term success but longterm failure. Plast Reconstr Surg 2002; 110: 940-1.

7. Gierloff M, Stöhring C, Buder T, et al. Aging changes of the midfacial fat compartments: a computed tomographic study. Plast Reconstr Surg 2012; 129: 263-73.

8. Ramanadham SR, Rohrich RJ. Newer understanding of specific anatomic targets in the aging face as applied to injectables: superficial and deep facial fat compartments: an evolving target for site-specific facial augmentation. Plast Reconstr Surg 2015; 136 (5 Suppl): 49S-55S.

9. Wan D, Amirlak B, Giessler P, et al. The differing adipocyte morphologies of deep versus superficial midfacial fat compartments: a cadaveric study. Plast Reconstr Surg 2014; 133: 615e-22e.

10. Donofrio LM. Fat distribution: a morphologic study of the aging face. Dermatol Surg 2000; 26: 1107-12.

11. Gosain AK, Klein MH, Sudhakar PV, et al. A volumetric analysis of soft-tissue changes in the aging midface using high resolution MRI: implications for facial rejuvenation. Plast Reconstr Surg 2005; 115: 1143-55.

12. Rohrich RJ, Ghavami A, Lemmon JA, et al. The individualized component face lift: developing a systematic approach to facial rejuvenation. Plast Reconstr Surg 2009; 123: 1050-63.

13. Rohrich RJ, Ghavami A, Constantine FC, et al. Lift-and-fill face lift: integrating the fat compartments. Plast Reconstr Surg 2014; 133: 756e-67e.

14. Bucky LP, Kanchwala SK. The role of autologous fat and alternative fillers in the aging face. Plast Reconstr Surg 2007; 120 (Suppl): 89S-97S.

15. Gosain AK, Klein MH, Sudhakar PV, Prost RW. A volumetric analysis of soft-tissue changes in the aging midface using high-resolution MRI: implications for facial rejuvenation. Plast Reconstr Surg 2005; 115: 1143-52.

16. Watanabe M, Buch K, Fujita A, et al. MR relaxometry for the facial ageing assessment: the preliminary study of the age dependency in the MR relaxometry parameters with- 
in the facial soft tissue. Dentomaxillofac Radiol 2015; 44: 20150047.

17. Ophir J, Cespedes I, Ponnekanti H, et al. Elastography: a quantitative method for imaging the elasticity of biological tissues. Ultrason Imaging 1991; 13: 111-34.

18. Drakonaki EE, Allen GM, Wilson DJ. Ultrasound elastography for musculoskeletal applications. Br J Radiol 2012; 85: 1435-45.

19. Bamber J, Cosgrove D, Dietrich CF, et al. EFSUMB guidelines and recommendations on the clinical use of ultrasound elastography. Part 1: basic principles and technology. Ultraschall Med 2013; 34: 169-84.

20. Xiang X, Yan F, Yang Y, et al. Quantitative assessment of healthy skin elasticity: reliability and feasibility of shear wave elastography. Ultrasound Med Biol 2017; 43: 445-52.

21. Cosgrove D, Piscaglia F, Bamber J, et al. EFSUMB guidelines and recommendations on the clinical use of ultrasound elastography. Part 2: clinical applications. Ultraschall Med 2013; 34: 238-53.

22. Alfageme Roldán F. Elastography in dermatology. Actas Dermosifiliogr 2016; 107: 652-60.

23. Ambroziak M, Noszczyk B, Pietruski P, et al. Elastography reference values of facial skin elasticity. Adv Dermatol Allergol 2018. https://doi.org/10.5114/ada.2018.77502

24. Ambroziak M, Pietruski P, Noszczyk B, Paluch Ł. Ultrasonographic elastography in the evaluation of normal and pathological skin - a review. Adv Dermatol Allergol 2018. doi: 10.5114/ada.2018.77069.

25. Ghassemi A, Prescher A, Riediger D, Axer H. Anatomy of the SMAS revisited. Aesthetic Plast Surg 2003; 27: 258-64.

26. Sun K, Kusminski CM, Scherer PE. Adipose tissue remodeling and obesity. J Clin Invest 2011; 121: 2094-101.

27. Kruglikov IL. General theory of body contouring: 2 . Modulation of mechanical properties of subcutaneous fat tissue. JCDSA 2014; 4: 117-27.

28. Fligiel SE, Varani J, Datta SC, et al. Collagen degradation in aged/photodamaged skin in vivo and after exposure to matrix metalloproteinase-1 in vitro. J Invest Dermatol 2003; 120: 842-8.

29. Sherratt MJ. Body mass index and dermal remodelling. Exp Dermatol 2015; 24: 922-3.

30. Ezure T, Amano S. Increment of subcutaneous adipose tissue is associated with decrease of elastic fibres in the dermal layer. Exp Dermatol 2015; 24: 924-9.

31. Loukas M, Kapos T, Louis RG, et al. Gross anatomical, CT and MRI analyses of the buccal fat pad with special emphasis on volumetric variations. Surg Radiol Anat 2006; 28: 254-60. 